\title{
THE JUNE MEETING IN BERKELEY
}

The two hundred seventieth regular meeting of the Society was held at the University of California on Thursday and Friday, June 20-21, 1929, in conjunction with the June 19-22 meetings of the American Association for the Advancement of Science. Sessions were held Thursday morning, Thursday afternoon, and Friday morning. The attendance included the following thirty-three members:

Alderton, J. P. Ballantine, Bateman, Bernstein, Biggerstaff, Cajori, Coleman, Corbin, Daus, Doole, G. C. Edwards, Garver, J. L. Gibson, W. L. Hart, M. W. Haskell, E. R. Hedrick, Hicks, Jerbert, D. N. Lehmer, Lenzen, Leonard, S. H. Levy, McFarlan, F. R. Morris, Pierpont, T. M. Putnam, J. M. Rankin, Pauline Sperry, Morgan Ward, G. T. Whyburn, W. M. Whyburn, A. R. Williams, B. C. Wong.

There was no meeting of the Council or of the Trustees of the Society.

The meeting was presided over at various times by President Hedrick and Professors M. W. Haskell, W. L. Hart James Pierpont, and Harry Bateman.

A luncheon for members and their guests was held at the Women's Faculty Club between the Thursday sessions.

At the Thursday morning session, by invitation of the Program Committee, Professor James Pierpont, of Yale University, delivered an address entitled Non-euclidean geometry in retrospect. The address will appear in full in a later issue of this Bulletin.

Titles and abstracts of other papers read at the meeting follow. The papers of the following authors were read by title: Clifford Bell, E. T. Bell, Brown, Cook, Craig, Hollcroft, Jones, Milne, Pierce, Roberts, Robertson, Rutt, Weyl, G. T. Whyburn (second paper), W. M. Whyburn (third paper), Wilder, Woodard, Zippin.

1. Professor J. P. Ballantine: $A$ vector derivation of Cramer's rule. 
A system of two equations in two unknowns may be written $\alpha x+\beta y=\gamma$, where $\alpha=\left(a_{1}, a_{2}\right)$ and similarly $\beta, \gamma$ are vectors. It is easily seen from a diagram that $x$ is the ratio between the altitudes on the common side $\beta$, and hence between the areas of the parallelograms $\gamma \beta$ and $\alpha \beta$. Hence the determinantal formula. The procedure generalizes readily to any number of dimensions. The paper will appear in the American Mathematical Monthly.

\section{Proiessor Harry Bateman: Some properties of spheroidal} harmonics.

The harmonics of even order associated with an oblate spheroid are expressed as definite integrals involving the corresponding harmonics for a prolate spheroid and some other interesting relations are obtained.

\section{Dr. Clifford Bell: Polygons jointly related to the rational} cubics and other curves.

A study is made of the $n$-sided polygon having $n-1$ of its vertices on, and all its sides tangent to, a rational cubic, the remaining vertex lying on any one of a finite set of algebraic curves $f_{i}(x, y, z)=0$ of orders $m_{i}$. The number of such $n$-sided polygons so connected with the cuspidal cubic is $3 \sum_{i=1}^{s} m_{i}$ where $s$ is the total number of curves $f_{i}(x, y, z)=0$. The number for the nodal cases is given by $3 \cdot 2^{n-1} \sum_{i=1}^{8} m_{i}$. The vertices of the polygons are ordered, and in the cuspidal case it is shown that by a simple magnification of $x, y$, and $z$, dependent only on $n$ and the affix of the vertices, any one of the curves of the set $f_{i}(x, y, z)=0$ can be deformed in such a way as to contain all of the vertices of given affix belonging to polygons associated with this curve and the given cubic. The well known results for triangles in and circumscribed to the rational cubics are obtained by specializing to the case $n=3$ and allowing the curves $f_{i}(x, y, z)=0$ to become coincident with the cubic.

\section{Professor E. T. Bell: Theorems on total representations} as sums of squares or triangular numbers.

If $m, n$ are coprime integers $>0$, and $f(x)$ is uniform and finite for integer values of $x, f(x)$ is said to be factorable if $f(m n)=f(m) f(n)$. Denote by $\mathrm{O}(n), E(n)$ the total numbers of representations of $n$ as sums of an odd, an even number of squares of integers $\gtrless 0$. Neither of $O(n), E(n)$ is factorable. In a former note (American Mathematical Monthly, vol. 30 (1923), p. 441), it was shown that $O(2 n+1)>E(2 n+1), E(2 n)>O(2 n)$ for all $n>0$. Here this property is widely generalized. As a very special case, $(-1)^{n-1}\left[\sum(-1)^{j} Q_{j}(n) / j\right]$ where $Q_{j}(n)=$ twice the number of representations of $n$ as a sum of $j$ squares with integer roots $\neq 0$, is factorable, where $\sum$ refers to $j=1,2, \cdots, n$; the sums of the positive, negative terms respectively satisfy the same inequalities as $E, O$. Further, the number of representations may be replaced by any even function of the actual integers in the respective representations, and the corresponding theorems then stated. The like is carried out for representations as sums of positive 
triangular numbers. The results are scarcely obvious by purely arithmetical methods; by the means used in the proofs given here, the theorems may be extended indefinitely.

5. Professor E. T. Bell: Non-existence theorems on the number of representations of arbitrary odd integers as sums of $4 r$ squares.

This paper appears in full in the present number of this Bulletin.

6. Professor E. T. Bell: Existence theorems on the number of representations of odd integers as sums of $4 t+2$ squares.

The number $N(n, 2 r)$ of representations of $n$ as a sum of $2 r$ squares, consists of two parts: first, a polynomial in the real divisors of $n$; second, a function which, for $n$ general, is not reducible to a polynomial in the real divisors of $n$. But if $n$ be restricted to be a member of certain arithmetic progressions, the second part may vanish identically. In the preceding paper, the necessary and sufficient conditions that this be so for $N(m, 4 r)$, $m$ odd, are determined. A remarkable transcendental equation in $r$ is the decisive condition. This paper closes, negatively, a reasonable conjecture arising from the last section of Eisenstein's classic paper, Crelle's Journal, vol. 34 (1847), p. 35. In order that $N(m, 4 r+2), m \equiv 3 \bmod 4$, $r>2$, shall be a polynomial in the real divisors of $m$, it is necessary (but not sufficient) that

$$
105\left(7^{2 r}-1\right)=\left(512 r^{4}-1280 r^{3}+1120 r^{2}+440 r-162\right)\left(3^{2 r}-1\right) .
$$

A similar condition holds for $m \equiv 1 \bmod 4$. Necessary and sufficient conditions, much more drastic, also are obtained, and thence the "divisor" character of $N(m, 4 r+2)$ is settled.

7. Professor E. T. Bell: On the representation of $4 t+2$ as a sum of $4 s$ squares.

See the preceding abstract.

8. Professor E. T. Bell: On the application of elliptic functions to universal and quasi-universal forms.

A form $f$ of degree 2 that represents all integers $>0$, or all integers $\geqq 0$, is called (after Dickson), universal. If $f$ represents all integers of a given type, that type to be finitely definable, $f$ is here called quasi-universal where $f$ is not universal. By the method of paraphrase much information concerning universal or quasi-universal forms can be obtained from extremely simple analysis. By the same method an immediate extension to forms of degree $>2$ is attainable. The possibilities of this application of elliptic functions being unlimited, only a selection is given, sufficient to show the method.

9. Professor B. A. Bernstein: On Whitehead and Russell's theory of deduction. 
Whitehead and Russell in their Principia Mathematica develop a theory of deduction as basis for the entire structure of the Principia. The present author discusses the nature of this theory and its relation to Boolean algebra.

\section{Professor B. A. Bernstein: Application of Boolean algebra to proving consistency and independence of postulates.}

The author shows how the problem of determining the consistency or the independence of a set of postulates can often be reduced to the solvable problem of determining the simultaneity of a set of Boolean relations.

11. Mr. A. B. Brown: Relations between the critical points of a real analytic function of $n$ independent variables.

Morse's paper Relations between the critical points of a real function of $n$ independent variables (Transactions of this Society, vol. 27, 1925) treats the case of non-degenerate critical points, that is, where the Hessian of the function $f$ is not zero at any critical point. In the present paper the author investigates the case in which this restriction is dropped, but requires $f$ to be analytic and have isolated critical points. Given a critical point at which $f=c$, not a point of minimum or maximum, a small $(n-1)$-sphere with center at the critical point is taken, and a corresponding small constant $e>0$. The $k$ th type number of the critical point, $k=1,2, \cdots, n-1$, is defined as the $(k-1)$ st order connectivity number, diminished by unity, of the complex of points of the locus $f=c-e$ within or on the sphere. Under this count Morse's relations are found to hold.

\section{Professor Florian Cajori: Generalizations in geometry} as seen in the history of developable surfaces.

During the period of primitive intuition in the study of surfaces, only cones and cylinders were known to be developable. There followed three stages of generalization: (1) by the application of infinitesimal analysis to real surfaces, the discovery of other real developable surfaces, as a special type of ruled surfaces; (2) by the freer use, in analysis, of complex numbers, the discovery of developable imaginary surfaces; (3) by transcending the limitations of infinitesimal analysis, the discovery of real developable surfaces which are not ruled.

13. Professor Florian Cajori: New data on the origin and spread of the dollar mark.

West Indian manuscripts of 1760 and 1778 link the $\$$ unmistakably with the Mexican sign $p^{s}$ for pesos or piastres, the $s$ being brought down upon the $p$. Nineteenth century manuscripts of Lower California furnish cumulative evidence in support of this origin. The symbol $\$$ spread from the West Indies and New Orleans to New York, the Mississippi region, and to other places in commercial relations with the West Indies and Gulf Coast. 
14. Professor Florian Cajori: Absurdities due to division by zero. A historical note.

Bishop Berkeley declared division by zero "shocking to good sense." The first to prove rigorously that division by zero is inadmissible was Martin Ohm. The first openly to derive absurdities from division by zero was Bernard Bolzano. The first to list the derivation of such absurdities as a mathematical recreation was W. W. R. Ball.

\section{Professor A. J. Cook: Pairs of rectilinear congruences with generators in one-to-one correspondence.}

When corresponding generators of a pair of rectilinear congruences do not intersect, a general theory for the projective study of the configuration may be constructed from a system of linear partial homogeneous differental equations of the first order in four dependent and two independent variables. The theory seems to be well adapted for the study of certain correspondences of Fubini, and a complete basis is furnished for their further investigation. The relation of Fubini's work to the earlier work of Darboux is also fully established. In connection with these correspondences a generalization to surfaces is secured for the concept of intersector curve which Lane introduced in his study of pairs of ruled surfaces.

16. Mr. H. V. Craig: On the simultaneous invariants of two functions.

This paper contains an investigation of the simultaneous invariants of two functions $F\left(x, x^{\prime}, x^{\prime \prime}\right)$ and $H\left(x, x^{\prime}\right)$. The chief result is a theory of parallelism developed from a fundamental tensor whose components are functions of $x, x^{\prime}$, and $x^{\prime \prime}$. The magnitude of vectors and the cosine of the angle between vectors undergoing parallel displacement are constant.

17. Professor P. H. Daus: Note on the solution of $x^{3}+2=y^{2}$.

The solutions of (1) $x^{3}+2=y^{2}$ in integers can be obtained from those of (2) $\alpha^{3}+3 \alpha \beta^{2}-2 \beta^{3}=1$ and conversely. The fact that (2) has solutions implies that the units in the cubic domain defined by $\theta^{3}+3 \theta-2=0$ have the form $x+\theta y+\theta^{2} z$, with $x=X^{2}+3 Y^{2}, y=X Y, z=Y^{2}$. The units in the cubic domain are connected by the recursion formulas $s_{n}=3\left(x_{1}-2 z_{1}\right) s_{n-1}$ $-3\left(x_{-1}-2 z_{-1}\right) s_{n-2}+s_{n-3}$, where $\left(x_{1}, y_{1}, z_{1}\right)$ is the fundamental unit and $\left(x_{-1}, y_{-1}, z_{-1}\right)$ its reciprocal. Since $s_{1}=(1,-1,-1)$, these recursion formulas can be used to prove (2) has no solution other than the trivial one $\alpha=1, \beta=0$, and (1) has only the solution $x=-1, y= \pm 1$. Several proofs of this fact have recently been given by other methods. The method outlined here may be extended to other equations of the type $x^{3}+a=y^{2}$.

18. Mr. H. P. Doole: Certain multiple-parameter expansions.

This paper deals with the proof of the convergence of the expansion of an arbitrary function in terms of the solutions of certain multiple-parameter 
systems of linear differential equations of the first order in two variables. In $\S 2$ the solutions of $X_{i}{ }^{\prime}+\left[\lambda a_{i 1}\left(x_{i}\right) \pm \mu a_{i 2}\left(x_{i}\right)\right] X_{i}=0,(i=1,2)$, are used with the boundary conditions $X_{i}\left(a_{i}\right)=\gamma_{i} X_{i}\left(b_{i}\right), \gamma_{i} \neq 0$. In $\S 3$ the system $X_{i}{ }^{\prime}+\left(\lambda x_{i}{ }^{h_{i}} \pm \mu\right) X_{i}=0, X_{i}(-a)=X_{i}(b),(i=1,2)$, has particular interest in the fact that $x_{i}{ }^{h_{i}}$ changes sign in the interval considered. Convergence is shown for the case where $h_{i}$ is integral, and possible divergence when $h_{i}$ is such that $x_{i}{ }^{h_{i}}$ is complex. In $\S 4$ convergence is proved for a certain system which has one auxiliary condition at more than two points of the interval, namely: $X(-1)-2 \alpha X(0)-X(1)=0$. In $\S \S 2-3$, the number of parameters might have been extended to $n$.

\section{Professor Raymond Garver: On the Brioschi normal} quintic.

The most direct reduction of a quintic equation to the Brioschi normal form seems to be that given by Gordan in volume 28 of the Mathematische Annalen, and improved successively by Weber and Dickson. However, the transformation which effects the reduction is valid only when a rather large number of restrictions is imposed on the given quintic. The present paper considers the significance of these restrictions (the matter has apparently not been considered before), and supplies valid transformations for two types of quintics to which the Gordan transformation cannot be applied.

20. Professor W. L. Hart: Limited trilinear forms in Hilbert space.

If a point $x\left(x_{1}, x_{2}, \cdots\right)$ lies in real Hilbert space, then $\left(\sum_{1} x_{i}{ }^{2}\right)^{1 / 2}$ will be called the modulus of $x$. The author considers real trilinear forms $A(x, y, z)=\sum_{i, j, k=1}^{\infty} a_{i j k} x_{i} y_{j} z_{k}$ where $(x, y, z)$ are in $H$-space. By definition, $A$ is limited if, for some $K>0,\left|\sum_{i, j, k=1}^{n} a_{i j k} x_{i} y_{j} z_{k}\right| \leqq K$ for all $n$ and for all $(x, y, z)$ whose moduli are at most 1 . It is proved that if, for any specified order of summation, $A(x, y, z)$ converges for all $(x, y, z)$ in $H$-space, then $A(x, y, z)$ is uniformly bounded for all $(x, y, z)$ whose moduli are at most 1 , and hence $A$ is limited. The method used in proving this theorem would apply as well to the proof of the uniform finiteness property for multilinear forms of any multiplicity. By use of familiar methods, it is proved that, if $A$ is limited, then its formal series converges either as a triple series or as an iterated sum, for each possible choice as to the order of summation among the indices $(i, j, k)$. Demonstrations are also given for other easily anticipated properties of limited trilinear forms.

\section{Professor M. W. Haskell: The cylindroid in non- euclidean geometry.}

The cylindroid is the locus of the axes of a pencil of linear complexes. In euclidean geometry this is a surface of the third order and of the third class. In non-euclidean geometry, however, a linear complex will have a pair of axes, which are mutually polar with respect to the absolute, and the locus of the axes of a pencil is a surface of the fourth order and fourth class. We shall use rectangular coordinates, so that the equation of the absolute 
is $x^{2}+y^{2}+z^{2}+k^{2} t^{2}=0$. If the special complexes of the pencil are real, their axes may be taken symmetrically as the lines

$$
y-m x=0, \quad z-c t=0 ; \quad y+m x=0, \quad z+c t=0 .
$$

The equation of the cylindroid is then, in point-coordinates,

$$
c\left(m^{2}+1\right) x y\left(z^{2} / k^{2}+t^{2}\right)=m\left(1+c^{2} / k^{2}\right) z t\left(x^{2}+y^{2}\right)
$$

and, in plane coordinates,

$$
c\left(m^{2}+1\right) \xi \eta\left(h^{2}+\theta^{2} / k^{2}\right)=m\left(1+c^{2} / k^{2}\right) h \theta\left(\xi^{2}+\eta^{2}\right),
$$

which, as $k=\infty$, reduce to the well known equations of the euclidean geometry. The surface is invariant under four infinite sets of collineations and is also autopolar with respect to the four pencils of quadric surfaces $x^{2}+y^{2}+2 \lambda z t=0, \quad x y+\lambda z t=0, \quad x^{2}+y^{2}+\lambda\left(z^{2}+k^{2} t^{2}\right)=0, \quad x y+\lambda\left(z^{2}+k^{2} t^{2}\right)=0$. The cylindroid can be shown to be the intersection of two linear complexes and of a quadratic complex.

\section{Professor E. R. Hedrick: Generalizations of Cauchy's} integral theorem.

In this paper, the author indicates generalizations of the well known Cauchy integral theorem for non-analytic functions of a complex variable.

23. Professor T. R. Hollcroft: Multiple points of algebraic curves.

In 1750, Cramer determined the maximum number of multiple points that may occur on algebraic curves of orders up to and including eight. In 1839, Plücker derived a general limit to the number of double points of an algebraic curve of given order. For multiple points of orders higher than two, limits have been found only in the case of individual curves. In the present paper, general limits are obtained defining the maximum number of multiple points of given order that may belong to an algebraic curve of given order. Two limits are found. When the order of the multiple point lies within a certain range of values, one of these limits applies; otherwise, the other.

\section{Professor Glenn James: Generalizations of Pascal's and} Brianchon's theorems.

Considering, in the case of Pascal's Theorem, that five vertices of a given hexagon are fixed, this paper studies the locus of the sixth vertex when the opposite sides of the hexagon are not necessarily collinear but various restrictions have been placed upon the triangle which they form. For example, if the determinant of the equations, (in cartesian coordinates), of the intersections of opposite sides of the hexagon is equal to a constant, the vertices of the hexagon describe twelve conics which reduce to the single conic on the vertices of the hexagon when the given constant is zero.

25. Dr. B. W. Jones: On two regular positive quadratic ternary forms. 
Previous to the appearance of this paper there has been no proof, as far as the author knows, that the form $x^{2}+8 y^{2}+32 z^{2}$ represents all positive integers of the form $8 n+1$ and that $x^{2}+2 y^{2}+32 z^{2}$ represents all positive integers of the forms $8 n+1$ and $8 n+3$. The Dirichlet method does not apply in this connection. Here, using some results derived by elliptic functions together with congruence theory, these proofs are given, thus completing the proof of the regularity of these two forms.

26. Professor L. H. McFarlan: Sets of necessary and sufficient conditions for the most simple problem of the calculus of variations.

Bolza in his Vorlesungen über Variationsrechnung establishes a set of five necessary conditions on an arc joining two fixed points of the plane, which minimizes an integral of the form $\int f(x, y, p) d x, p=d y / d x$. He also gives a set of sufficient conditions on such an arc in order that it should have the above property. These sets are not identical. In his treatment of the corresponding problem in the parametric form one set of conditions is given which is shown to be both necessary and sufficient in order that the arc have the minimizing property. In some respects the two problems treated may be shown to differ. For example the minimizing arc in the non-parametric form of the problem must not have a tangent parallel to the $y$ axis. Such a restriction is removed in the parametric treatment of the problem. There exists however a class of problems which may be treated by either method. The existence of a set of necessary and sufficient conditions on the minimizing arc in the parametric problem indicates the existence of one such set in the other form. The problem is studied with the intention of closing the apparent gap existing between the set of necessary and the set of sufficient conditions in the non-parametric form of the problem by transforming the single set of conditions in the parametric problem into the non-parametric form.

27. Professor L. H. McFarlan: Sufficient conditions in the problem of Lagrange with one variable end point.

In the problem of Lagrange one considers an integral $\int f\left(x, y_{1}, \cdots, y_{n}\right.$, $\left.y_{1}{ }^{\prime}, \cdots, y_{n}{ }^{\prime}\right) d x$ and a set of differential equations $\phi_{\alpha}\left(x, y_{1}, \cdots, y_{n}\right.$, $\left.y_{1}{ }^{\prime}, \cdots, y_{n}{ }^{\prime}\right)=0,(\alpha=1, \cdots, m<n)$. The functions $y_{1}, \cdots, y_{n}$ are to satisfy the differential equations $\phi_{\alpha}=0$ and to render the integral an extreme value. In this paper the values of the functions $y_{1}(x), \cdots, y_{n}(x)$ are considered fixed for $x_{1}$, while the values for $x_{2}$ are restricted by a set of equations $x_{2}=X(t), y_{12}=Y_{1}(t), \cdots, y_{n 2}=Y_{n}(t)$. In this paper is given a set of sufficient conditions which when satisfied by the functions $y_{1}(x), \cdots, y_{n}(x)$ will cause them to minimize the above integral and satisfy the set of differential equations.

28. Professor L. H. McFarlan: Problems of the calculus of variations in several dependent variables and their derivatives of various orders. 
The problem of minimizing an integral whose integrand contains $x$ and $n$ dependent variables $y_{i}(i=1, \cdots, n)$ and their derivatives $y_{i}{ }^{\prime}, y_{i}{ }^{(2)}, \cdots, y_{i}{ }^{\left(k_{i}\right)},\left(k_{i} \geqq 0\right)$, is treated by means of the known theory of the problem of Lagrange in the calculus of variations. Necessary and sufficient conditions on the minimizing arc analogous to those of Legendre, Jacobi, and Weierstrass in the more simple problems are derived. These are readily seen to contain as special cases the results obtained by Forsyth (Calculus of Variations, 1927) in which one dependent variable and derivatives of the first and second order enter the integrand. In the most simple problem of the calculus of variations with one dependent variable and its derivative of the first order, one passes from the fixed end-point case to problems in which the end points are required to lie on certain curves in the plane. In this paper the values of the functions $y_{i}$ and their derivatives up to and including those of order $k_{i}-1$ for the values $x_{1}$ and $x_{2}$ defining the end points of the minimizing arc, are restricted by a system of differential equations of order $k_{i}-1$ in the variables $y_{i}$. The number of these differential equations is expressible in terms of $n$ and the numbers $k_{i}$. For this case one obtains a necessary condition corresponding to the usual transversality condition. For special choice of the differential equations the focal point property is also generalized.

29. Professor W. E. Milne: On the degree of convergence of the Gram-Charlier series.

In a previous communication the author has given certain sufficient conditions for an assigned degree of convergence of the series

$$
f(x)=\sum_{m=0}^{n} c_{m} e^{x / 4}\left[\left(d / d x^{m}\right) e^{-x^{2} / 2}\right]+R_{m}(x) .
$$

It is now shown that the following conditions are necessary: if $R_{n}(x)$ $=O\left(n^{-k-\epsilon}\right)$ for every $n$, where $k$ is an integer and $\epsilon>0$, then $f(x)$ must have a continuous derivative of order $2 k$; if $R_{n}(x)=O\left(n^{-k}\right)$ uniformly in the infinite interval, then $f(x)=O\left(x^{-2 k}\right)$ when $x$ is large. It is also shown that if $f(x)$ has a $(k-1)$ th derivative satisfying a Lipschitz condition and if $f(x)$ and its derivatives vanish to a suitable order at infinity, then the constants $c_{m}$ can be chosen so that $R_{n}(x)=O\left(n^{-k / 2}\right)$.

30. Professor T. A. Pierce: On an algorithm and its use in approximating roots of algebraic equations.

If in the generating equations of a continued fraction the operation of addition be replaced by multiplication and that of division into unity be replaced by subtraction from unity we obtain a new algorithm. The expansion by this algorithm of any rational number is periodic. Non-periodic expansions represent irrational numbers. The application of the algorithm in approximating roots of algebraic equations is simple.

31. Mr. J. H. Roberts: Upper semi-continuous collections of continua some of which are unbounded.

In an abstract of a paper presented to the Society on December 27, 1928, 
I failed to mention a certain hypothesis in the statement of certain results. The corrected statement is as follows: "If $G$ is an upper semi-continuous collection of continua no one of which separates the plane $S$ and such that (1) every point of the plane $S$ belongs to some continuum of $G,(2)$ at least two continua of $G$ are unbounded, and (3) the space of elements of $G$ is metric, then the space of elements of $G$ is in continuous one-to-one correspondence with a subset of the plane $S$. In the present paper the condition that no continuum of $G$ separates $S$ is removed and the space of elements of $G$ is shown to be a subset of a cactoid. A cactoid-as defined by R. L. Moore-is a bounded continuous curve $M$ lying in space of three dimensions and such that (a) every non-degenerate maximal cyclic subset of $M$ is a simple closed surface and (b) no point of $M$ lies in a bounded complementary domain of any subcontinuum of $M$.

32. Mr. J. H. Roberts: On a problem of Menger concerning regular curves.

In 1927 Menger raised the question as to whether or not, for every positive number $e$, every regular curve $M$ is the sum of a finite number of continua of diameter less than $e$, the common part of any two of which is at most one point. In the present paper an example is given which shows that the answer to Menger's question is in the negative. It is proved that for the special case where the set of points of $M$ of order greater than 2 is null-dimensional Menger's question can be answered in the affirmative

33. Professors H. P. Robertson and Hermann Weyl: On a problem in the theory of groups arising in the foundations of infinitesimal geometry.

The paper appears in full in the present number of this Bulletin.

34. Dr. N. E. Rutt: On the structure of certain plane continua.

Let $x$ denote a point and $Z$ a bounded plane continuum which is the sum of a set $\left[X_{\alpha}\right]$ of continua each of which contains the point $x$, each of which remains connected when $x$ is subtracted from it, and no two of which have any point in common except $x$. If none of the continua of $\left[X_{\alpha}\right]$ has a bounded complementary domain containing a point of any other continuum of the set $\left[X_{\alpha}\right]$, order is easily assigned among the elements of $\left[X_{\alpha}\right]$, so that with respect to some reference element precedence of elements of $\left[X_{\alpha}\right]$ and clockwise sequences may be defined. The following facts concerning the relations possible among the continua of $\left[X_{\alpha}\right]$ are established. If $\left[X_{i}\right]$ is a clockwise sequence of elements of $\left[X_{\alpha}\right]$, then no element $X_{a}$ of $\left[X_{\alpha}\right]$ preceding all the elements of $\left[X_{i}\right]$ contains any limit point of $\left[X_{i}\right]$ except $x$. If $\left[X_{i}\right]$ is a clockwise sequence of elements of $\left[X_{\alpha}\right]$, and if both $X_{a}$ and $X_{b}$ are elements of $\left[X_{\alpha}\right]$ preceding all the elements of $\left[X_{i}\right]$, then of the two prime ends of $X_{a}+X_{b}$ containing $x$ which are arc-wise accessible from the unbounded complementary domain of $X_{a}+X_{b}$ the one which is not a limit of any element of $\left[X_{i}\right]$ is also not a limit of the set of elements $\left[X_{i}\right]$. 


\section{Dr. Morgan Ward: The foundations of general arith-} metic.

This paper is the first part of an amplification and extension of two short notes on the same topic (Proceedings of the National Academy, vol. 13 (1927), pp. 748-749; vol. 14 (1928), pp. 907-911). By taking as a basis a set closed under logical multiplication instead of a semi-group closed under ordinary multiplication, a postulational definition of an abstract arithmetic is obtained which insures unique factorization whether the set contains a finite or an infinite number of elements. Furthermore all the classical arithmetics (e.g., ideals of an algebraic field) are instances of this abstract system. A detailed development of the formal properties of the system discloses many analogies with the Boolean algebra of logic. In fact, every finite arithmetic may be interpreted as a set of elements in a Boolean algebra, although the converse theorem is false. Finally the case where the introduction of ideal elements is necessary is studied from the same abstract viewpoint.

\section{Professor Hermann Weyl: On the foundations of general infinitesimal geometry.}

The paper appears in full in the present number of this Bulletin.

37. Professor G. T. Whyburn: Note on simple closed curves.

In this paper it is shown that a simple closed curve (in $n$-space) may be defined as a compact continuum every point of which separates it locally into exactly two components; and in the plane it may be defined as a compact continuum every point of which is accessible from exactly two complementary domains. If these continua are not compact, they are open curves. It is also pointed out that if every point of a continuum $M$ separates it locally into exactly $k$ components, ( $k$ finite and $>1$ ), then $k=2$, and $M$ is a simple closed curve or an open curve according as it is or is not compact.

38. Professor G. T. Whyburn: On the set of all cut points of a continuous curve.

In this paper it is shown that as a consequence principally of the author's results on the structure of a continuous curve relative to its cyclic elements it follows that the set of all cut points of any continuous curve in a metric space is homeomorphic with a certain kind of subset of a plane acyclic continuous curve. It is also shown, conversely, that a subset of this kind of any acyclic continuous curve in a euclidean space of dimension $>1$ is always identically the set of all cut points of some continuous curve. Thus we obtain a complete characterization of a point set which is topologically equivalent to the set of all cut points of a continuous curve. Incidentally, the results of this paper answer a question raised by $C$. Zarankiewicz in the Bulletin de l'Académie Polonaise, 1926, p. 362.

39. Professor W. M. Whyburn: Functional properties of solutions of differential systems. 
In a recent paper (Annals of Mathematics, vol. 30 (1928), pp.31-39) the author established existence and uniqueness theorems for a general class of differential systems. Using the notation of that paper, the present paper interprets the notion of the solutions of the differential systems being continuous functionals of the functions $f_{i}\left(x, y_{1}, \cdots, y_{n}\right), A_{i j}\left(x, y_{1}, \cdots, y_{n}\right)$, $i, j=1, \cdots, n$, and proves that under the hypotheses of the above-mentioned existence theorems the solutions of the systems will be continuous functionals of this type. The results here include the well known theorem concerning the continuity of the solutions of linear systems as functionals of the coefficients. The results of the present paper are useful in a study of the effects produced by small perturbations on dynamical systems.

40. Professor W. M. Whyburn: On the critical sets for functions of $n$ real variables.

In a former paper, (Non-isolated critical points of functions, which appears in the present issue of this Bulletin (701-708), the author defined crit$i c a l$ sets for functions of $n$ independent real variables in cases where the critical points of these functions were not isolated. Minimal sets were characterized and theorems concerning the distribution of these were proved. The present paper continues this classification of critical sets to define several additional classes and extends the former theorems to cover this classification. The definitions and extensions are shown to be consistent with the classification by Morse (Transactions of this Society, vol. 27 (1925), pp. 345-396) when the critical points are isolated.

\section{Professor W. M. Whyburn: Functional inequalities.}

Inequalities of the types $F(x)<\sum_{i=1}^{n} f_{i}(x) g_{i}(x), F(x) \leqq \sum_{i=1}^{n} f_{i}(x) g_{i}(x)$ where the functions involved are non-negative and continuous on the open or closed, finite or infinite interval $X$ of the real variable $x$, are studied with a view to determining continuous functions $h_{i}(x)$ such that $0 \leqq h_{i}(x) \leqq g_{i}(x)$, $F(x)=\sum_{i=1}^{n} f_{i}(x) g_{i}(x)$ on $X$, and these functions take on assigned boundary values at points of given subsets of $X$. Cases where measurability and summability replace continuity in the above statements are treated. Functions of $m$ real independent variables are considered. Applications to proofs of properties of differential equations are given.

42. Professor R. L. Wilder: Characterizations of continuous curves that are perfectly continuous.

It was shown by R. L. Moore and the author (see this Bulletin, vol. 29, p. 294) that in order that a continuum $M$ should be a continuous curve, it is necessary and sufficient that every component of an open subset of $M$ should be arcwise connected. The present paper considers those continuous curves which have the property that all of their subcontinua are continuous curves-such curves are called, for the sake of brevity, perfectly continuous, or perfect continuous curves. It is shown that the above property characterizing continuous curves may be modified so as to characterize perfect continuous curves. If $M$ is any point set, and $K$ is an $F_{\sigma}$ which is a subset 
of $M$, then let us call $M-K$ a quasi-open subset of $M$. Then in order that a continuum $M$ in $E_{n}$ should be a perfect continuous curve, it is necessary and sufficient that every component of a quasi-open subset of $M$ should be arcwise connected. Incidentally, it is shown that every connected subset of a perfect continuous curve in a locally compact metric space is connected im kleinen-a property which also obviously characterizes these curves.

\section{Dr. A. R. Williams: On a type of transformation con- nected with a pencil of curves of order $n$.}

The fact that the $n^{2}$ base points of a pencil of curves of order $n$ are determined by $n(n+3) / 2-1$ of them gives rise to transformations of the plane. It is the purpose of this paper to discuss the locus of the remaining base points when a given number of them are fixed and the others necessary to determine the pencil are taken consecutive on some curve. Use is made of rational surfaces and developables in space of 3 and higher dimensions.

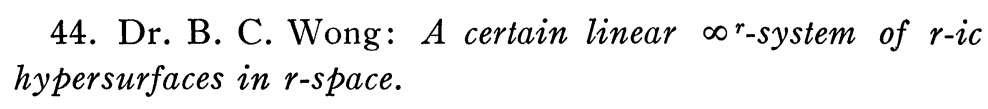

The hypersurfaces of order $r$ passing through $r+1$ given $(r-2)$-spaces in $S_{r}$ form a linear $\infty{ }^{r}$-system $|V|$. The composite base manifold of $|V|$ is of order $r(r+1) / 2$ and is composed of the $r+1$ given, $(r-2)$-spaces and the locus of the $\infty^{r-3}$ lines incident with them. This locus is of order $(r+1)(r-2) / 2$. It is shown that any $r$ hypersurfaces of $|V|$ meet in only one distinct point and that, therefore, each hypersurface can be represented rationally on a hyperplane of $S_{r}$. It is possible to find $r$ hyperquadric surfaces that will effect the transformation by making any point and the point of intersection of its polar hyperplanes with respect to the $r$ hyperquadric surfaces correspond to each other.

45. Dr. B. C. Wong: An octavic hypersurface in space of four dimensions.

This paper deals with the hypersurface $V_{r-1}$ of order $2^{r-1}$ in $r$-space represented by the equation $x_{0}^{1 / 2}+x_{1}^{1 / 2}+\cdots+x_{r}^{1 / 2}=0$ and, in particular, with the octavic hypersurface $V_{3}{ }^{8}$ in 4 -space. $V_{r-1}$ is the generalization to $r$-space of Steiner's quartic surface in 3-space. It is touched by each of the $\left(\begin{array}{c}r-1 \\ k+1\end{array}\right) k$-spaces $[0<k<r]$ of the coordinate simplex along $2^{r-k-1}$ coincident $(k-1)$-dimensional varieties of order $2^{k-1}$. The representation of $V_{3}^{8}$ and that of its reciprocal hypersurface on a hyperplane are discussed and also that of its general hyperplane section.

46. Professor D. W. Woodard: On a system of axioms for two-dimensional analysis situs.

In the author's doctoral dissertation, On two-dimensional analysis situs with special reference to the Jordan curve theorem, published in volume 13 of Fundamenta Mathematicae, the following axiom is used: If $P$ is a point on the boundary of a region $R$, there exist two simple continuous arcs $A P$ and 
$B P$ such that $A P-P$ and $B P-P$ are subsets of $R$ and $S-R^{\prime}$ respectively. Here $S$ represents the set of all points in the space under consideration and $R^{\prime}$, the set of points contained in $R$ and its derivative. It is proved that the axiom stated above may be replaced by the following weaker axiom: Every point on the boundary of a region is a limit point of the exterior of the region. In connection with this axiom an independence example is provided. All of the results of the paper follow from the modified system of axioms. In fact, it is necessary only to make certain additional arguments in the proof of Theorem 7 of the paper.

\section{Dr. Leo Zippin: On acyclic continuous curves.}

The author constructs a plane, bounded, acyclic continuous curve which has a subset homeomorphic with any given acyclic continuous curve. In case the given curve is unbounded it is homeomorphic with the complement in a bounded acyclic continuous curve of a subset of end points. He proves, also, that if $B$ is a closed and totally disconnected subset of a continuous curve $C$, in order that $B$ be the set of end points of an acyclic continuous subcurve of $C$ it is necessary and sufficient that if $M$ is any bounded continuous curve of $C$ and $D$ a closed and totally disconnected subset of interior points of $M$, then $M-D$ is connected.

B. A. Bernstein

Associate Secretary 